\title{
Evaluation of the safety and immunogenicity in United Kingdom laboratory workers of a combined Haemophilus influenzae type $b$ and meningococcal capsular group $C$ conjugate vaccine
}

Jamie Findlow ${ }^{1 *}$, Helen Findlow ${ }^{1}$, Sarah Frankland ${ }^{1}$, Ann Holland ${ }^{1}$, Daniel Holme1, Emma Newton ${ }^{1}$, Jo Southern², Pauline Waight ${ }^{2}$, Ed Kaczmarski ${ }^{1}$, Elizabeth Miller ${ }^{2}$ and Ray Borrow ${ }^{1,3}$

\begin{abstract}
Background: Although a combined Haemophilus influenzae type b (Hib)/meningococcal capsular group C (MenC) conjugate vaccine with a tetanus toxoid carrier protein (Hib/MenC-TT) is not licensed for use in those above 2 years of age due to lack of data on safety and efficacy, certain patient groups at high risk of MenC and/or Hib disease are recommended to receive it. Laboratory workers working with Hib and/or MenC cultures may be at a potentially increased risk of acquiring infectious diseases and vaccination is therefore an important safety consideration. We undertook a clinical trial to investigate the safety and immunogenicity of Hib/MenC- $T$ vaccine in this cohort.
\end{abstract}

Methods: A total of 33 subjects were recruited to the trial, all of whom were vaccinated. Serology was completed on samples taken at baseline and four weeks following vaccination to determine MenC specific IgG, MenC serum bactericidal antibody (SBA), anti-Hib polyribosylribitol phosphate (PRP) IgG and anti-tetanus toxoid lgG responses.

Results: At baseline, high proportions of subjects had protective antibody concentrations against MenC, Hib and tetanus due to previous vaccination and/or natural exposure. Vaccination induced > 3, 10 and 220 fold increases in geometric mean concentrations for MenC SBA, anti-tetanus toxoid IgG and anti-Hib PRP IgG, respectively. Following vaccination, $97 \%$ of subjects had putative protective SBA titres $\geq 8,100 \%$ had short term protective anti-Hib PRP IgG concentrations $\geq 0.15 \mu \mathrm{g} / \mathrm{mL}$ and $97 \%$ had protective anti-tetanus toxoid concentrations $\geq 0.1 \mathrm{IU} / \mathrm{mL}$. No safety concerns were reported with minor local reactions being reported by $21 \%$ of subjects.

Conclusions: Immunological responses determined in this trial are likely a combination of primary and secondary responses due to previous vaccination and natural exposure. Subjects were a representative cross-section of laboratory workers, enabling us to conclude that a single dose of Hib/MenC-TT was safe and immunogenic in healthy adults providing the evidence that this vaccine may be used for providing protection in an occupational setting.

Keywords: Meningococcal, Haemophilus influenzae type b, Tetanus, Vaccine, Laboratory workers, Occupational immunisation

\footnotetext{
* Correspondence: jamie.findlow@phe.gov.uk

'Public Health England, Public Health Laboratory, Manchester, Manchester Medical Microbiology Partnership, PO Box 209, Clinical Sciences Building II, Manchester Royal Infirmary, Manchester M13 9WZ, UK

Full list of author information is available at the end of the article
} 


\section{Background}

Glycoconjugate vaccines to provide protection against Haemophilus influenzae type b (Hib) and Neisseria meningitidis capsular group $\mathrm{C}(\mathrm{MenC})$ were implemented into the UK immunisation schedule in 1992 and 1999, respectively $[1,2]$. The Hib vaccine was extremely effective in the targeted age group reducing invasive disease in England and Wales from almost 500 cases per year to 20 cases, two years following implementation [1]. The MenC vaccine was similarly successful and reduced disease incidence by $86.7 \%$ in the targeted age groups, also within two years of implementation [3]. Despite the success of these vaccines, cases in the UK general population still occur from which live isolates are initially cultured in local microbiology laboratories prior to transfer to reference laboratories at Public Health England (PHE). Transmission of $\mathrm{Hib}$ and MenC is achieved via the aerosol/respiratory route and as laboratory staff handle live cultures they can be considered to have a potential occupational exposure. Use of live cultures is not restricted to clinical and reference laboratories as there are many additional laboratories undertaking research. This results in a significant population of laboratory workers with a potential exposure risk including those undertaking functional immunoassays such as the MenC and Hib serum bactericidal antibody (SBA) assay to evaluate vaccine responses.

The potential risk was confirmed in an analysis conducted in the UK which determined laboratory workers to have a 184-fold increased risk of meningococcal disease compared to the general population [4]. This supports the requirement for employers to provide protection wherever possible to laboratory staff, with a potential occupational exposure to infectious disease [5]. Protection from acquisition and disease in the laboratory should primarily rely on physical control measures, however occupational vaccination is an important final form of defence. This is highlighted by a number of reports of potentially vaccinepreventable meningococcal cases in laboratory staff [6-10].

Occupational vaccination in the UK against meningococcal disease over the last decade has been generally achieved using monovalent MenC conjugate (MCC), A and $\mathrm{C}$ bivalent polysaccharide and quadrivalent $\mathrm{A}, \mathrm{C}, \mathrm{Y}$ and $\mathrm{W}$ vaccines initially in the form of polysaccharide formulations which have now been superseded by conjugate products [11]. Vaccination against Hib has been more problematic as the only available vaccines are combination vaccines designed for infant immunisation. In 2005, Menitorix ${ }^{\ominus}$ a combined $\mathrm{Hib} / \mathrm{MenC}$ conjugate vaccine with a tetanus toxoid (TT) carrier protein was licensed in Europe and incorporated into the UK immunisation schedule from September 2006 as a 12 month booster vaccination [1]. The vaccine is also licensed for primary vaccination in infants from 2 months up to 12 months of age as a three dose course given with an interval of at least 1 month between doses. Although not licensed or generally for use in children above 2 years of age due to lack of data on safety and efficacy, it is recommended in certain patient groups to reduce the number of immunisations required [11]. Children and adults with asplenia or splenic dysfunction, may have a suboptimal response to MCC vaccine [12] and are recommended to receive a single dose of Hib/MenC-TT followed one month later by a single dose of a quadrivalent meningococcal A, C, Y and W conjugate vaccine [11].

The availability of Hib/MenC-TT vaccine provided the opportunity to offer vaccination to laboratory staff who routinely work with live $\mathrm{Hib}$ and/or MenC cultures at the Manchester Medical Microbiology Partnership (MMMP). We therefore undertook a clinical trial to evaluate the immunogenicity and safety of a single dose of Hib/MenC-TT vaccine in staff at a potential occupational exposure to $\mathrm{Hib}$ and/or MenC.

\section{Methods}

\section{Study population and schedule}

Enrolment into this single dose Hib/MenC-TT vaccine trial was open to adult ( $\geq 18$ years of age) laboratory staff from the MMMP who were considered to be at potential occupational exposure to Hib and/or MenC. Potential occupational exposure was defined as routine handling of live Hib and/or MenC cultures which included both scientific staff from the PHE Meningococcal Reference Unit and the Vaccine Evaluation Unit (VEU) and porters/autoclave staff. At the time of enrolment this incorporated a greater range of staff than were at that time required to receive occupational meningococcal vaccination which was limited to scientific staff. Inclusion criteria ensured participants had no contraindications to vaccination as specified in the "Green Book" [11], written informed consent and availability for at least the initial trial (vaccination) visit. To allow the vaccine to be provided to as many laboratory staff as possible, exclusion criteria were limited to known or suspected pregnancy.

The non-adjuvanted $\mathrm{Hib} / \mathrm{MenC}-\mathrm{TT}$ vaccine $(0.5 \mathrm{~mL}$ dose containing $5 \mu \mathrm{g}$ of Hib polysaccharide (polyribosylribitol phosphate (PRP)) conjugated to $12.5 \mu \mathrm{g}$ of TT and $5 \mu \mathrm{g}$ of MenC polysaccharide conjugated to $5 \mu \mathrm{g}$ of TT (GlaxoSmithKline, UK), from a single lot (Batch number A76CA008A), was administered in the deltoid muscle of the non-dominant arm. Vaccination was deferred if the oral temperature measured was $>38^{\circ} \mathrm{C}$, or if there was acute illness on the day of vaccination. Blood samples were taken before and 4 weeks following vaccination. Subjects were asked to provide details of any medication taken at enrolment and throughout the follow up period and meningococcal vaccine histories were obtained from the Occupational Health Department. 


\section{Serology}

Serum samples were assayed in the VEU, using a multiplexed fluorescent bead assay to quantify IgG antibody concentrations to MenC, Hib PRP and tetanus toxoid, based upon previously published methodology $[13,14]$. Functional antibody activity against MenC was determined in the SBA assay as previously described [15]. The target strain in the SBA assay was C11 (C:16:P1.7-1,1) and the complement source was baby rabbit sera (Pel-Freeze Incorporated, Rodgerson, AZ, USA) (rSBA). rSBA titres were expressed as the reciprocal of the final serum dilution giving $\geq 50 \%$ killing at 60 minutes. For computational purposes, rSBA titres lower than the serum starting dilution of 4 were assigned a value of 2 .

\section{Correlates of protection}

Immune correlates indicative of protection have been established for Hib, MenC and tetanus [16-18]. Hib PRP IgG concentrations $\geq 0.15 \mu \mathrm{g} / \mathrm{mL}$ and $\geq 1.00 \mu \mathrm{g} / \mathrm{mL}$ are considered the putative short term and long term protective levels, respectively [17]. For MenC, a rSBA titre of $\geq 8$, four weeks after vaccination was shown to predict short term clinical protection against MenC disease in the UK [16] while a rSBA titre $\geq 128$ reliably predicted a SBA titre using human complement of $\geq 4$ [19], the accepted hSBA correlate of protection [20,21]. For tetanus, anti-toxoid levels $<0.1 \mathrm{IU} / \mathrm{mL}$ denote susceptibility and levels $\geq 1.0 \mathrm{IU} / \mathrm{mL}$ are considered as providing long term protection $[18,22]$.

\section{Safety}

Subjects were monitored for 30 minutes following vaccination and then completed a daily health diary for seven days recording any local reactions (erythema, swelling, tenderness) and measurements if present. Oral temperature was also recorded as well as any visits to a doctor, hospital or general practitioner or any medication taken. A vaccine research nurse followed up subjects by telephone 24 hours following vaccination to enquire about any reactions or problems.

\section{Analyses}

At each time point the proportions of subjects achieving rSBA titres $\geq 8$ and $\geq 128$, anti-PRP IgG antibody concentrations $\geq 0.15 \mu \mathrm{g} / \mathrm{mL}$ and $\geq 1.00 \mu \mathrm{g} / \mathrm{mL}$ and anti-tetanus toxoid IgG antibody concentrations $\geq 0.10 \mathrm{IU} / \mathrm{mL}$ and $\geq$ $1.00 \mathrm{IU} / \mathrm{mL}$ were calculated. MenC rSBA geometric mean titres (GMTs) and MenC-specific IgG, anti-PRP IgG and anti-tetanus toxoid IgG geometric mean concentrations (GMCs) with 95\% confidence intervals (95\% CI) were also calculated. Due to the small sample sizes, immunogenicity analysis did not include any formal statistical analysis.

\section{Governance}

The trial was conducted in accordance with the 1996 International Conference on Harmonisation Good Clinical Practice (ICH GCP) guidelines, the 2000 Declaration of Helsinki and the 2004 EU Clinical Trial Directive. A favorable opinion was given by the UK Medicines and Healthcare products Regulatory Agency (MHRA) and the National Research Ethics Service, Northern and Yorkshire Research Ethics Committee. The EudraCT number was 2006-004302-74 and the trial was registered on the public website, www.clinicaltrials. gov under the identifier: NCT00503165.

\section{Results}

A total of 35 staff responded to trial advertisements of which 33 were available for the first visit and were enrolled and vaccinated (Figure 1). Women accounted for $61 \%(20 / 33)$ of those enrolled reflecting the higher proportion of women laboratory staff within the eligible recruitment cohort. At enrolment the mean age of subjects was 30 years 2 months (range, 22 years 5 months to 59 years 10 months). Of the 33 subjects vaccinated, blood samples were obtained for 29 subjects at both time points. Three

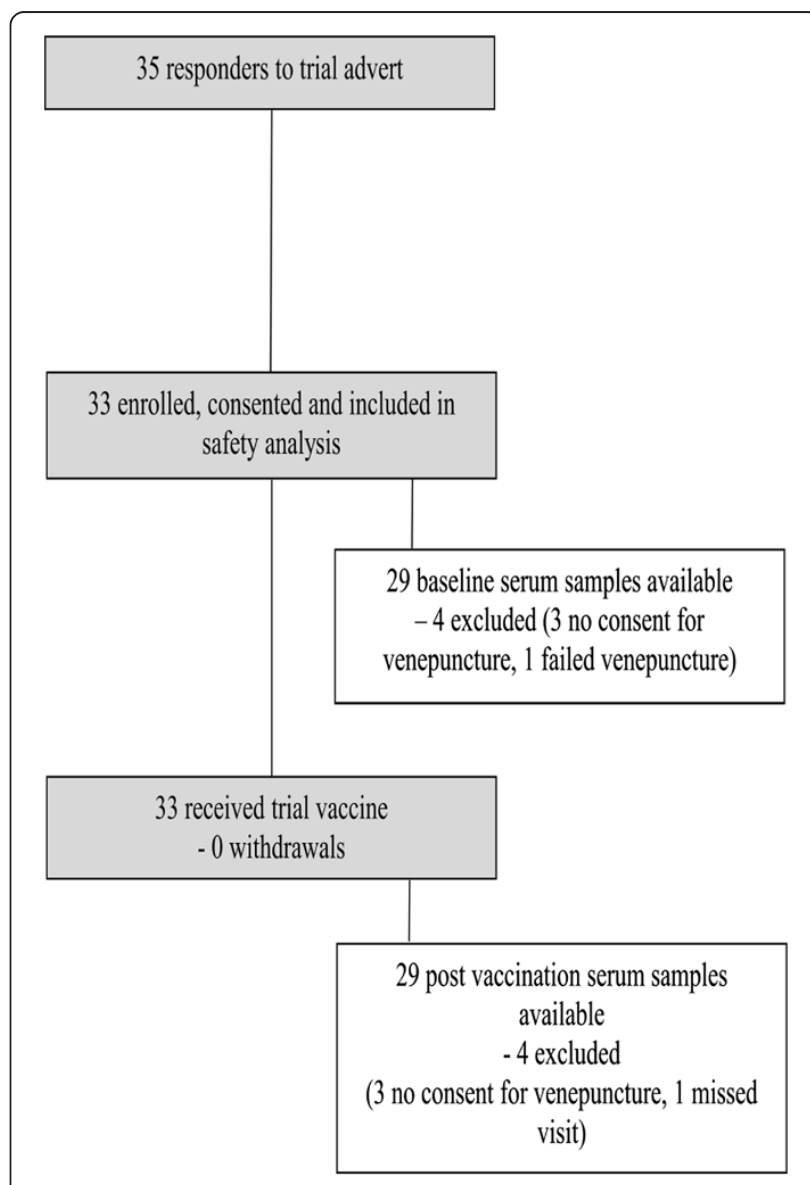

Figure 1 Participant flow chart. 
subjects did not consent to venepuncture but were still enrolled and vaccinated as the investigators' principal aim was to afford potential benefit of vaccination to as many staff as possible. The trial was undertaken between July and September 2007 and the mean number of days between the baseline blood draw/vaccination and blood draw following vaccination sample was 31 days (range 18-64 days; median 28 days). Telephone follow-up at 24 hours was completed for all participants and diaries were completed and returned by 29 subjects.

\section{Immunogenicity}

Prior to vaccination, $79 \%$ and $66 \%$ of subjects had putative protective MenC rSBA titres $\geq 8$ and at the higher cut off of $\geq 128$, respectively (Table 1 ). Following vaccination, a 3 fold increase in MenC rSBA GMT was determined whilst MenC-specific IgG GMC only increased 1.5 fold (Table 1). Proportions of subjects with rSBA titres above the two cut offs increased from the initially high background levels with all but one subject achieving an rSBA titre $\geq 8$ following vaccination (Table 1 ).

For $\mathrm{Hib}$ at baseline, high proportions of subjects (62\%) had anti-PRP IgG $\geq 0.15 \mu \mathrm{g} / \mathrm{mL}$, the putative short term protective concentration while a lower proportion $(31 \%)$ had putative long term protective concentrations $\geq$ $1.0 \mu \mathrm{g} / \mathrm{mL}$ (Table 1). Following vaccination, a $>200$ fold increase in anti-PRP IgG GMC was determined which resulted in all subjects achieving anti-PRP concentrations $\geq 0.15 \mu \mathrm{g} / \mathrm{mL}$ and all but one achieving concentrations $\geq 1.0 \mu \mathrm{g} / \mathrm{mL}$ (Table 1 ).

Anti-tetanus toxoid IgG serology undertaken to determine responses induced by the carrier protein indicated a $>10$ fold increase in IgG GMC following vaccination in comparison to baseline levels (Table 2). This resulted in all but one subject achieving anti-tetanus toxoid IgG concentrations $\geq 0.10 \mathrm{IU} / \mathrm{mL}$ and $\geq 1.0 \mathrm{IU} / \mathrm{mL}$ compared to $90 \%$ and $45 \%$ at baseline, respectively (Table 2 ).

\section{Safety}

Concomitant medication was reported by 11 (33\%) participants. Two participants (6\%) took over the counter tablets - one took Ibuprofen for an exercise injury on day 0 of the trial, and the other took paracetemol on day 8 of the trial for a raised temperature and nasal congestion. Nine participants (27\%) took medication for chronic conditions during the trial, which the trial medic assessed as unrelated to trial treatment.

From the 29 diaries returned, six participants (21\%) reported local reactions following vaccination, of which tenderness was most frequently reported (Table 3). Five participants (17\%) reported systemic symptoms (Table 3). Participants reporting hay fever, hypertension and nasal congestion/raised temperature were medicated as above. In one participant an episode of dizziness and nausea commenced approximately 8 hours following vaccination and subsided after 48 hours. The individuals with hypertension and hay fever consulted their GPs about these conditions. There were no visits to hospital, or Serious Adverse Events reported during the trial.

\section{Discussion}

To our knowledge this trial is the first report of the safety and immunogenicity of a Hib/MenC-TT combination vaccine in healthy adults which to date, has only been approved for children from 2 months up to 2 years of age. The vaccine was safe and immunogenic in the trial population indicating that it is suitable for use in an occupational setting to provide protection to laboratory staff which may have a potential occupational exposure to Hib and/or MenC.

Investigation of the immunogenicity of the MenC vaccine component was complicated as trial subjects were laboratory workers, who had previously received meningococcal vaccination. Consequently, $79 \%$ of subjects had baseline MenC rSBA titres $\geq 8$ which is at least double, than

Table 1 Proportions of subjects with MenC serum bactericidal antibody titres $\geq 8 / \geq 128$, MenC serum bactericidal antibody geometric mean titres with $95 \%$ confidence intervals, anti-MenC IgG geometric mean concentration with 95\% confidence intervals, anti-PRP Hib IgG geometric mean concentration with $\mathbf{9 5 \%}$ confidence intervals and proportions of subjects with anti-PRP Hib IgG $\geq 0.15 / \geq 1.00 \mu \mathrm{g} / \mathrm{mL}$ before and following vaccination with Hib/MenC-TT

\begin{tabular}{lcc}
\hline & Baseline & Following vaccination \\
\hline rSBA GMT $(95 \%$ Cl) & $151.2(52.2-437.9)$ & $454.3(200.9-1027.3)$ \\
$N(\%)$ with rSBA titres $\geq 8$ & $23 / 29(79 \%)$ & $28 / 29(97 \%)$ \\
$N(\%)$ with rSBA titres $\geq 128$ & $19 / 29(66 \%)$ & $24 / 29(83 \%)$ \\
MenC IgG GMC $(95 \% \mathrm{Cl}) \mu \mathrm{g} / \mathrm{mL}$ & $4.3(2.1-8.8)$ & $6.6(3.3-13.1)$ \\
N (\%) with anti-PRP Hib lgG concentrations $\geq 0.15 \mu \mathrm{g} / \mathrm{mL}$ & $18 / 29(62 \%)$ & $29 / 29(100 \%)$ \\
N (\%) with anti-PRP Hib IgG concentrations $\geq 1.00 \mu \mathrm{g} / \mathrm{mL}$ & $9 / 29(31 \%)$ & $28 / 29(97 \%)$ \\
Anti-PRP Hib IgG GMC $(95 \% \mathrm{Cl}) \mu \mathrm{g} / \mathrm{mL}$ & $0.23(0.09-0.60)$ & $50.69(24.28-105.84)$ \\
\hline
\end{tabular}


Table 2 Proportions of subjects with anti-tetanus toxoid IgG antibody concentrations $\geq 0.10 / 1.0 \mathrm{IU} / \mathrm{mL}$ and geometric mean concentrations with $95 \%$ confidence intervals at baseline and following vaccination with Hib/MenC-TT

\begin{tabular}{lrr}
\hline & \multicolumn{1}{c}{ Baseline } & Following vaccination \\
\hline $\mathrm{N}(\%)$ with anti-tetanus toxoid lgG concentrations $\geq 0.10 \mathrm{IU} / \mathrm{mL}$ & $26 / 29(90 \%)$ & $28 / 29(97 \%)$ \\
$\mathrm{N}(\%)$ with anti-tetanus toxoid lgG concentrations $\geq 1.00 \mathrm{IU} / \mathrm{mL}$ & $13 / 29(45 \%)$ & $28 / 29(97 \%)$ \\
Anti-tetanus toxoid GMC $(95 \% \mathrm{Cl}) \mathrm{IU} / \mathrm{mL}$ & $0.77(0.47-1.27)$ & $8.20(4.65-14.46)$ \\
\hline
\end{tabular}

previously reported in the UK general population $\geq 25$ years of age in 2009 [23]. At enrolment, subjects with prior vaccination against MenC had been vaccinated with $\mathrm{MCC}$ vaccine either through the MCC catch-up campaign during 1999 to 2000 [2], a MCC staff trial [24] and/or through the Occupational Health Department with multivalent polysaccharide vaccines. Eight subjects had also received 3 doses of the "Norwegian" outer membrane vesicle (OMV) vaccine, MenBvac (Norwegian Institute of Public Health), in a trial in 2005/6 [25]. The OMV vaccine may have been responsible for elevated baseline rSBA titres due to induction of cross-reactive antibodies against subcapsular proteins on the MenC target strain. Of the six staff without putative protective MenC rSBA titres, three were not required to be vaccinated by the then current occupational vaccination program although two had received MCC vaccination seven years previously. The three other staff had received a quadrivalent polysaccharide vaccine three years previously and were two years away from the recommended five year re-vaccination point [11]. The clinical significance of SBA titres below the protective level is undetermined as while SBA titres $\geq$ than the protective level equate to protection from disease, a SBA titre < than the protective level does not necessarily indicate susceptibility $[20,21]$.

Following vaccination, all but one subject achieved MenC rSBA titres $\geq 8$ and the MenC rSBA GMT increased three fold from baseline levels. The rSBA response was mainly achieved in subjects with low baseline titres as the average individual fold change in rSBA titre following vaccination was 226.0 and 1.6 in subjects with baseline

Table 3 Local and systemic reactions reported by subjects following vaccination

Local reactions

\begin{tabular}{lc} 
Any & $6 / 29(21 \%)$ \\
Tenderness & $4 / 29(14 \%)$ \\
Erythema & $2 / 29(7 \%)$ \\
& Systemic reactions \\
Any & $5 / 29(17 \%)$ \\
Headache & $1 / 29(3 \%)$ \\
Nausea/dizziness & $1 / 29(3 \%)$ \\
Hypertension & $1 / 29(3 \%)$ \\
Nasal congestion/raised temperature & $1 / 29(3 \%)$ \\
Hay fever & $1 / 29(3 \%)$ \\
\hline
\end{tabular}

rSBA titres $<128$ and $\geq 128$, respectively (data not presented). This is in agreement with previous studies where negative correlations between baseline antibody concentrations and subsequent booster responses have been reported, including trials using Hib/MenC-TT as a booster [26].

The phenomenon of immunological hyporesponsiveness, characterised by decreased immunological responses upon repeated doses of MenC containing vaccines after initial MenC polysaccharide vaccination has been well documented in both adults [27-30] and younger children [31-33]. Two previous laboratory staff studies utilising MCC vaccine also demonstrated hyporesponsiveness in staff who had previously been vaccinated with meningococcal A and $\mathrm{C}$ bivalent polysaccharide vaccine in comparison to naïve controls [24,34]. Unfortunately, we were not able to make the same comparison in our own trial as all but one of the subjects had previously received meningococcal vaccination. Interestingly, these two trials reported greater increases in rSBA GMT and IgG GMC in both the naïve and the previously vaccinated groups than achieved in the trial we report here. It is difficult to determine the reason for this although the most apparent differences were that the previous trials used MCC as opposed to Hib/MenC-TT and many of the subjects in our trial had more complex previous meningococcal vaccination histories with high baseline immunity against MenC. In the future, any detrimental impact of prior polysaccharide vaccination should hopefully become less of an issue as meningococcal quadrivalent conjugate vaccines are now available and are recommended over polysaccharide vaccines for use in at risk-groups [11].

Despite none of the subjects reporting previous vaccination against $\mathrm{Hib}, 62 \%$ and $31 \%$ of subjects had baseline anti-Hib PRP IgG levels consistent with short and long term protection, respectively. These proportions were similar, although higher than those reported in the UK general population in 2009, whereby $57 \%$ and $21 \%$ of the 25-44 year age group were reported to have short and long term protective anti-Hib PRP IgG levels, respectively [35]. Following vaccination a $>200$ fold rise in anti-PRP IgG GMC was measured, all subjects achieved short term protective antibody concentrations and all but one subject achieved an anti-PRP level $\geq 1.00 \mu \mathrm{g} / \mathrm{mL}$.

Subjects also had baseline tetanus toxoid IgG levels similar to that in the UK adult general population in 2009 [36] with $10 \%$ and $45 \%$ of subjects at baseline and 
$17 \%$ and $44 \%$ of the UK general population with susceptible and long term protective antibody concentrations, respectively. Following vaccination a $>10$ fold rise in IgG GMT was achieved and all but one subject seroconverted to achieve IgG levels over $0.1 \mathrm{IU} / \mathrm{mL}$. These data indicate that immune response to $\mathrm{Hib} / \mathrm{MenC}-\mathrm{TT}$ was not restricted to the two polysaccharide antigens and that the carrier protein is also able to induce a robust immune response in primed adults. Similar findings have previously been reported in meningococcal conjugate vaccines trials $[37,38]$ and is an important finding as laboratory workers are also recommended to receive appropriate tetanus vaccination [11]. The ability of $\mathrm{Hib} /$ MenC-TT to boost tetanus antibody concentrations may negate the need to use an additional specific tetanus containing vaccine. This is of particular benefit as the unavailability of a monovalent tetanus vaccine in Europe, requires the use of a combination vaccine (Revaxis ${ }^{\circ}$, Sanofi Pasteur MSD) which in laboratory workers potentially unnecessarily contains diphtheria and inactivated poliovirus antigens.

Following Hib/MenC-TT, it was three different individuals who failed to achieve protective antibody levels against $\mathrm{MenC}, \mathrm{Hib}$ and tetanus indicating that this was not due to a single individual/vaccination. Overall, the vaccine was well tolerated with a maximum of $21 \%$ of subjects reporting any systemic or local reactions of which they were generally minor and of short duration. This reactogenicity profile, is similar to that which would be predicted from previous $\mathrm{Hib} / \mathrm{MenC}-\mathrm{TT}$ trials undertaken in pediatric populations [39]. Unfortunately, it is not possible to compare reactogenicity to the one trial completed in older children as no safety data were reported [40].

\section{Conclusions}

In conclusion, $\mathrm{Hib} / \mathrm{MenC}-\mathrm{TT}$ when administered as a single dose to healthy adults is safe and immunogenic. Due to subject's previous meningococcal and tetanus vaccination in combination with probable natural exposure, immunological responses were likely to be a combination of primary and secondary responses. These are however, a representative sample of laboratory workers/healthy adults and therefore, provide evidence that this vaccine may be used in the occupational setting for providing protection against MenC and/or Hib. The vaccine also induced a robust response against the carrier protein, indicating that the vaccine can also boost protection against tetanus. The importance of these findings relates to the unavailability of monovalent conjugate Hib vaccines in Europe, necessitating the use of multivalent products of which Hib/MenC-TT has the lowest valency. These data reported here from healthy adults may also provide an insight into the immunogenicity and safety of Hib/MenC-TT in other at-risk populations, thus supporting the UK vaccination policy for vaccinating asplenic and hyposlpenic individuals [11].

\section{Competing interests}

All authors are employees of PHE. JF, HF, SF, AH, DH, EN and RB undertake contract research on behalf of PHE for pharmaceutical companies, including GlaxoSmithKline, Novartis, Sanofi Pasteur MSD, Baxter and Pfizer. JF has acted as a consultant on behalf of PHE and has received travel assistance from GlaxoSmithKline, Novartis, Baxter and Pfizer. HF has acted as a consultant on behalf of PHE and has received travel assistance from Baxter.

\section{Authors' contributions}

$\mathrm{RB}$ and $\mathrm{EM}$ conceived the trial. JF, RB, EM and EK designed the trial and EK was the Chief Investigator and trial medic. JF, HF, SF, AH, DH and EN were responsible for completion of immunoassays. JF, JS, PW, EK and RB were responsible for trial coordination, maintenance of regulatory compliance and safety monitoring. All authors read and approved the final manuscript.

\section{Acknowledgements}

The work was supported and sponsored by PHE (formerly the Health Protection Agency). We would also like to thank the staff who participated in this trial and the Vaccine Research Nurses, Lynne Joslin, Mary Heath and Nora Ashwood.

This is an independent trial funded by the Policy Research Programme in the Department of Health, United Kingdom, grant 039/031. The views expressed in the publication are those of the authors and not necessarily those of the Department of Health.

\section{Author details}

${ }^{1}$ Public Health England, Public Health Laboratory, Manchester, Manchester Medical Microbiology Partnership, PO Box 209, Clinical Sciences Building II, Manchester Royal Infirmary, Manchester M13 9WZ, UK. ${ }^{2}$ Immunisation Department, Health Protection Services, Public Health England, Colindale, London NW9 5EQ, UK. ${ }^{3}$ University of Manchester, Inflammation Sciences Research Group, School of Translational Medicine, Stopford Building, Manchester M13 9PL, UK.

Received: 28 January 2014 Accepted: 26 June 2014

Published: 16 July 2014

\section{References}

1. Ladhani SN: Two decades of experience with the Haemophilus influenzae serotype b conjugate vaccine in the United Kingdom. Clin Ther 2012, 34:385-399.

2. Miller E, Salisbury D, Ramsay M: Planning, registration, and implementation of an immunisation campaign against meningococcal serogroup C disease in the UK: a success story. Vaccine 2001, 20(Supplement 1):S58-S67.

3. Balmer $P$, Borrow $R$, Miller $E$ : Impact of meningococcal $C$ conjugate vaccine in the UK. J Med Microbiol 2002, 51:717-722.

4. Boutet R, Stuart JM, Kaczmarski EB, Gray SJ, Jones DM, Andrews N: Risk of laboratory-acquired meningococcal disease. J Hosp Infect 2001, 49:282-284.

5. Health and Safety Executive, Health Services Advisory Committee: Safe Working and the Prevention of Infection in Clinical Laboratories and Similar Facilities. London, UK: HSE Books; 2003.

6. Kessler AT, Stephens DS, Somani J: Laboratory-acquired serogroup A meningococcal meningitis. J Occup Health 2007, 49:399-401.

7. Athlin S, Vikerfors T, Fredlund HH, Olcén P: Atypical clinical presentation of laboratory-acquired meningococcal disease. Scand J Infect Dis 2007, 2007 (39):911-913.

8. Bhatti AR, DiNinno VL, Ashton FE, White LA: A laboratory-acquired infection with Neisseria meningitidis. J Infect 1982, 4:247-252.

9. Omer H, Rose G, Jolley KA, Frapy E, Zahar JR, Maiden MC, Bentley SD, Tinsley CR, Nassif X, Bille E: Genotypic and phenotypic modifications of Neisseria meningitidis after an accidental human passage. PLoS One 2011, 6:e17145. 10.1371/journal.pone.0017145

10. From the Centers for Disease Control and Prevention: Laboratory-acquired meningococcal disease-United States, 2000. JAMA 2002, 287:1256-1258.

11. Department of Health: Immunisation against infectious disease - 'the green book'. http://immunisation.dh.gov.uk/category/the-green-book/. 
12. Balmer $\mathrm{P}$, Falconer $\mathrm{M}, \mathrm{McD}$ onald $\mathrm{P}$, Andrews $\mathrm{N}$, Fuller E, Riley C, Kaczmarski $E$, Borrow $R$ : Immune response to meningococcal serogroup $C$ conjugate vaccination in asplenic individuals. Infect Immun 2004, 72:332-337.

13. Lal G, Balmer P, Joseph H, Dawson M, Borrow R: Development and evaluation of a tetraplex flow cytometric assay for quantitation of serum antibodies to Neisseria meningitidis serogroups A, C, Y, and W-135. Clin Diagn Lab Immunol 2004, 11:272-279.

14. Pickering JW, Martins TB, Schroder MC, Hill HR: Comparison of a multiplex flow cytometric assay with enzyme-linked immunosorbent assay for quantitation of antibodies to tetanus, diphtheria, and Haemophilus influenzae type b. Clin Diagn Lab Immunol 2002, 9:872-876.

15. Maslanka SE, Gheesling LL, LiButti DE, Donaldson KBJ, Harakeh HS, Dykes JK, Arhin FF, Devi SJN, Frasch CE, Huang JC, Kris-Kuzemenska P, Lemmon RD, Lorange M, Peeters CCAM, Quataert S, Tai JY, Carlone GM: Standardization and a multilaboratory comparison of Neisseria meningitidis serogroup A and C serum bactericidal assays. Clin Diag Lab Immunol 1997, 4:156-167.

16. Andrews N, Borrow R, Miller E: Validation of serological correlate of protection for meningococcal $C$ conjugate vaccine using efficacy estimates from post-licensure surveillance in England. Clin Diag Lab Immunol 2003, 10:780-786.

17. Kayhty $H$, Peltola $H$, Karanko $V$, Makela PH: The protective level of serum antibodies to the capsular polysaccharide of Haemophilus influenzae type b. J Infect Dis 1983, 147:1100.

18. Borrow R, Balmer P, Roper MH: The Immunological Basis for Immunisation Series. Module 3: Tetanus. Geneva, Switzerland: World Health Organization; 2007.

19. Borrow R, Andrews N, Goldblatt D, Miller E: Serological basis for use of meningococcal serogroup $C$ conjugate vaccines in the United Kingdom: a re-evaluation of correlates of protection. Infect Immun 2001, 69:1568-1573.

20. Goldschneider I, Gotschlich EC, Artenstein MS: Human immunity to the meningococcus: I. The role of humoral antibodies. J Exp Med 1969, 129:1307-1326.

21. Goldschneider I, Gotschlich EC, Artenstein MS: Human immunity to the meningococcus: II. Development of natural immunity. J Exp Med 1969, 129:1327-1348.

22. Maple PA, Jones CS, Wall EC, Vyse A, Edmunds WJ, Andrews NJ, Miller E: Immunity to diphtheria and tetanus in England and Wales. Vaccine 2000, 19:167-173.

23. Ishola DA, Borrow R, Findlow H, Findlow J, Trotter C, Ramsay ME: Prevalence of serum bactericidal antibody to serogroup $C$ Neisseria meningitidis in England a decade after vaccine introduction. Clin Vaccine Immunol 2012, 19:1126-1130.

24. Borrow R, Southern J, Andrews N, Peake N, Rahim R, Acuna M, Martin S, Miller E, Kaczmarski E: Comparison of antibody kinetics following meningococcal serogroup $C$ conjugate vaccine between healthy adults previously vaccinated with meningococcal $A / C$ polysaccharide vaccine and vaccine-naïve controls. Vaccine 2001, 19:2050-3043.

25. Findlow J, Taylor S, Aase A, Horton R, Heyderman R, Southern J, Andrews N, Barchha R, Harrison E, Lowe A, Boxer E, Heaton C, Balmer P, Kaczmarski E, Oster P, Gorringe A, Borrow R, Miller E: Comparison and correlation of Neisseria meningitidis serogroup $B$ immunological assays and human antibody responses following three doses of the 'Norwegian' meningococcal outer membrane vesicle vaccine, MenBvac. Infect Immun 2006, 74:4557-4565

26. Blanchard-Rohner G, Watt H, Kelly DF, Yu LM, Snape MD, Pollard AJ: Baseline polysaccharide-specific antibodies may not consistently inhibit booster antibody responses in infants to a serogroup $C$ meningococcal protein-polysaccharide conjugate vaccine. Vaccine 2012, 30:4153-4159.

27. Jokhdar H, Borrow R, Sultan A, Adi M, Riley C, Fuller E, Baxter D: Immunological hyporesponsiveness to group $C$ but not group $A$ following repeated meningococcal $\mathrm{A} / \mathrm{C}$ polysaccharide vaccination in Saudi Arabia. Clin Diagn Lab Immunol 2004, 11:83-88.

28. Keyserling H, Papa T, Koranyi K, Ryall R, Bassily E, Bybel MJ, Sullivan K, Gilment G, Reinhardt A: Safety, immunogenicity and immune memory of a novel meningococcal (groups A, C, Y and W135) polysaccharide diptheria toxoid conjugate vaccine (MCV-4) in health adolescents. Arch Pediatr Adolesc Med 2005, 159:907-913.

29. Granoff DM, Gupta RK, Belshe RB, Anderson EL: Induction of immunological refractoriness in adults by meningococcal $C$ polysaccharide vaccination. J Infect Dis 1998, 178:870-874.
30. Richmond P, Kaczmarski E, Borrow R, Findlow J, Clark S, McCann R, Hill J, Barker M, Miller E: Meningococcal C polysaccharide vaccine induces immunological hyporesponsiveness in adults that is overcome by meningococcal C conjugate vaccine. J Infect Dis 2000, 181:761-764.

31. Gold R, Lepow ML, Goldschneider I, Draper T, Gotschlich EC: Clinical evaluation of group $A$ and $C$ meningococcal polysaccharide vaccine in infants. J Clin Invest 1975, 56:1536-1547.

32. MacDonald NE, Halperin SA, Law BJ, Forrest B, Danzig L, Granoff DM: Induction of immunologic memory by conjugated vs. plain meningococcal C polysaccharide vaccine in toddlers. JAMA 1998, 280:1685-1689.

33. Findlow H, Sow S, Borrow R, Tapia M, Haidara FC, Akinsola AK, Idoko OT, Diallo F, Adegbola R, Tang Y, Parulekar V, Chadha H, Mabey L, Holme D, Townsend K, Chaumont J, LaForce FM, Kulkarni PA, Marchetti E, Viviani S, Hassan-King M, Preziosi MP: Meningococcal group C and W135 immunological hyporesponsiveness in African toddlers. Clin Vaccine Immunol 2011, 18:1492-1496.

34. Southern J, Deane S, Ashton L, Borrow R, Goldblatt D, Andrews N, Balmer P, Morris R, Kroll JS, Miller E: Effects of prior polysaccharide vaccination on magnitude, duration, and quality of immune responses to and safety profile of a meningococcal serogroup $\mathrm{C}$ tetanus toxoid conjugate vaccination in adults. Clin Diagn Lab Immunol 2004, 11:1100-1104.

35. Ladhani S, Ramsay M, Flood J, Campbell H, Slack M, Pebody R, Findlow J, Newton E, Wilding M, Warrington R, Crawford H, Min S, Gray K, Martin S, Frankland S, Bokuvha N, Laher G, Borrow R: Haemophilus influenzae serotype B (Hib) seroprevalence in England and Wales in 2009. Euro Surveill 2012, 17(46).

36. Wagner KS, White JM, Andrews N, Borrow R, Stanford E, Newton E, Pebody RG: Immunity to tetanus and diphtheria in the UK in 2009. Vaccine 2012, 30:7111-7117

37. Burrage M, Robinson A, Borrow R, Andrews N, Southern J, Findlow J, Martin S, Thornton C, Goldbaltt D, Corbel M, Sesardic D, Cartwright K, Richmond P, Miller E: Effect of vaccination with carrier protein on response to meningococcal $C$ conjugate vaccines and value of different immunoassays as predictors of protection. Infect Immun 2002, 70:4946-4954.

38. Kshirsagar N, Mur N, Thatte U, Gogtay N, Viviani S, Preziosi MP, Elie C, Findlow H, Carloen G, Borrow R, Parulekar V, Plikaytis B, Kulkarni P, Imbault N, LaForce M: Safety, immunogenicity, and antibody persistence of a new meningococcal group A conjugate vaccine in health Indian adults. Vaccine 2007, 25S:A101-A107.

39. Miller JM, Mesaros N, Van Der Wielen M, Baine Y: Conjugate meningococcal vaccines development: GSK Biologicals experience. Adv Prev Med 2011, 10.4061/2011/846756.

40. Perrett KP, Winter AP, Kibwana E, Jin C, John TM, Yu LM, Borrow R, Curtis N, Pollard AJ: Antibody persistence after serogroup $C$ meningococcal conjugate immunisation of United Kingdom primary-school children in 1999-2000 and responses to a booster: a phase 4 clinical trial. Clin Infect Dis 2010, 50:1601-1610.

\section{doi:10.1186/1745-6673-9-26}

Cite this article as: Findlow et al:: Evaluation of the safety and immunogenicity in United Kingdom laboratory workers of a combined Haemophilus influenzae type $\mathrm{b}$ and meningococcal capsular group $\mathrm{C}$ conjugate vaccine. Journal of Occupational Medicine and Toxicology 2014 9:26.

\section{Submit your next manuscript to BioMed Central and take full advantage of:}

- Convenient online submission

- Thorough peer review

- No space constraints or color figure charges

- Immediate publication on acceptance

- Inclusion in PubMed, CAS, Scopus and Google Scholar

- Research which is freely available for redistribution 